\title{
SINGLE SERVER QUEUEING NETWORKS WITH VARYING SERVICE TIMES AND RENEWAL INPUT
}

\author{
PIERRE LE GALL \\ France Telecom, RED \\ 4 Parc de la Bérengère \\ F-92210 Saint-Cloud, France
}

(Received January, 1999; Revised December, 1999)

Using recent results in tandem queues and queueing networks with renewal input, when successive service times of the same customer are varying (and when the busy periods are frequently not broken up in large networks), the local queueing delay of a single server queueing network is evaluated utilizing new concepts of virtual and actual delays (respectively). It appears that because of an important property, due to the underlying tandem queue effect, the usual queueing standards (related to long queues) cannot protect against significant overloads in the buffers due to some possible "agglutination phenomenon" (related to short queues). Usual network management methods and traffic simulation methods should be revised, and should monitor the partial traffic streams loads (and not only the server load).

Key words: Queueing Networks, Concentration Tree, Tandem Queue, Local Queueing Delay, Jitter Delay, Agglutination Phenomena, Apparent Queueing Delay, Buffer Overload.

AMS subject classifications: $60 \mathrm{~K} 25,90 \mathrm{~B} 22$.

\section{Introduction}

In this paper, we utilize recent results in tandem queues and queueing networks to evaluate the local queueing delay in single server queueing networks (excluding ring networks) with renewal input, when successive service times of the same customer are varying, and when busy periods are frequently not broken up in large networks (leading to the useful concept of equivalent tandem queue). We assume that customers only gain access to a downstream queue after completion of the upstream service. At each queue, the service discipline is "first-come-first served" (FC-FS).

Classical theories (i.e., the product form theory) ignore some queueing phenomena which occur between entry to the network and the considered local point, and influence the local queue. In particular, the influence of the indistinguishability of the 
customers inside the upstream busy periods affects the distribution towards a given destination. Markovian queueing networks typically use the concept of local transition rates independent of upstream interferences. For converging traffic streams, busy periods tend to amalgamate from state-to-stage, leading to a predominant influence of the longest service times. In the same way, this indistinguishability in the upstream busy periods leads to a new concept of a local apparent queueing delay related to the overall upstream queueing delay. Here, only a part of the delay observed upstream is perceived downstream, leading to some reduction factor for diverging traffic streams, and to new concepts of virtual and actual local queueing delays, respectively.

Curiously and due to this reduction factor, this last phenomenon of indistinguishability tends to assimilate the long local queue to a classical G/G/1 server queue, and the usual queueing standards may only use the $G / G / 1$ server model. This does not mean that the actual queueing model can be represented simply by a $G / G / 1$ server. On the contrary, it may be dangerous to dimension the buffers using only this $G / G / 1$ server model, particularly in case of infinite support for the service time distribution. In the network, servers are occupied by service time. But in the buffers, servers are occupied by sojourn time (i.e., the sum of the queueing delay and the service time). Short and medium service times may overload buffers due to the agglutination phenomenon of short service times behind long service times, leading to the same occupancy for long and short service times. Since busy periods tend to amalgamate, this phenomenon is amplified from stage-to-stage, leading to a strong influence of the longest service times with a necessarily limited length.

If the methods of buffer dimensioning have to be revised, the same follows fro network management methods in which partial traffic streams loads (i.e., traffic intensities) should be monitored (and not only the server loads). This avoids some fast buffer congestion generalizing (in a large area of the network) the inaccessibility to the servers, since the agglutination phenomenon is transmitted downstream immediately.

In Section 2, we define our notation and assumptions. In Section 3, we outline our earlier studies in tandem queues and queueing networks for the case of single servers with renewal input. In Section 4, we deduce the evaluation of the local queue distribution in single server queueing networks (with renewal input), with an application to buffer overload and buffer rejection rate. In Section 5 , we conduct a numerical study (with Poisson arrivals) for a single link packet switched network, in which the links handle three populations of packets (with constant packet lengths). Between these populations, packet lengths are very different, proving the strong impact of the longest packets on buffer overload and buffer rejection rate.

\section{Notation and Assumptions}

We assume the system is in the stationary regime.

\subsection{The Local Queue}

The total arrival process at a local queue (considered at the entry to the network) is assumed to be renewal, with $\boldsymbol{F}_{\mathbf{0}}(\boldsymbol{t})$ denoting the distribution function of the interarrival intervals. Local service times are mutually independent and independent of the 
arrival process. The local queueing delay is usually assimilated to the queueing delay $\boldsymbol{W}_{\mathbf{0}}$ of a GI/G/1 queue, with the following data for an arbitrary customer:

- $\quad$ arrival rate $\lambda$;

- $\quad$ local service time $T$;

- $\operatorname{Prob}(\boldsymbol{T}<t)=\boldsymbol{F}_{\mathbf{1}}(\boldsymbol{t})$

- local sojourn time $\boldsymbol{S}=W_{0}+T$

- $\quad$ overall upstream service time $\boldsymbol{T}^{\mathbf{\prime}}$;

- $\operatorname{load}(=$ traffic intensity $)=\rho=\lambda \cdot T$.

Note that the upstream service times are not necessarily independent of the downstream service times. We set for $\operatorname{Re}(z) \geq 0$ :

- $\varphi_{0}(z)=\int_{0}^{\infty} e^{-z t} \cdot d F_{0}(t)$

- $E e^{-z T}=\varphi_{1}(z)=\int_{0}^{\infty} e^{-z t} \cdot d F_{1}(t)$;

- $\quad E e^{-z W_{0}}=W_{0}(z)$;

- $\quad E e^{-z S}=\Phi_{1}(z)=W_{0}(z) \cdot \varphi_{1}(z)$.

To present the following expressions, we will use Cauchy contour integrals along the imaginary axis in the complex plane $\boldsymbol{u}$. If the contour (followed from the bottom to the top) is to be right of the imaginary axis (the contour being closed at infinity to the right), we write $\int+0$. If the contour is to the left of the imaginary axis, we write $\int_{-0}$. Pollaczek [6] gave the expressions:

$$
\begin{aligned}
& W_{0}(z)=\operatorname{Exp}\left\{\frac{-1}{2 \pi i} \int_{-0}\left[\frac{1}{z-u}+\frac{1}{u}\right] \cdot \log \left[1-\varphi_{0}(-u) \cdot \varphi_{1}(u)\right] \cdot d u\right\} \\
& \operatorname{Prob}\left(W_{0}=0\right)=Q_{1}=\operatorname{Exp}\left\{\frac{-1}{2 \pi i} \int_{-0} \log \left[1-\varphi_{0}(-u) \cdot \varphi_{1}(u)\right] \cdot \frac{d u}{u}\right\}
\end{aligned}
$$

For tandem queues, it will be useful to introduce these other expressions, related to the case where $T<t$ :

$$
\begin{gathered}
\varphi_{1}(z, t)=\int_{0}^{t} e^{-z \alpha} \cdot d F_{1}(\alpha) \\
Q_{1}(t)=\operatorname{Exp}\left\{\frac{-1}{2 \pi i} \cdot \int_{-0} \log \left[1-\varphi_{0}(-u) \cdot \varphi_{1}(u, t)\right] \cdot \frac{d u}{u}\right\} .
\end{gathered}
$$

Consequently, we have:

$$
Q_{1}=Q_{1}(\infty)
$$

\subsection{The Queueing Network}

In Figure 1, representing a full network, a number $\boldsymbol{n}$ of identical incoming paths of $\boldsymbol{m}$ successive servers are distributed [at stage $(m+1)$ ] upon a number $\boldsymbol{n}$ of final servers, defining the final stage. The traffic stream $\boldsymbol{A}_{\boldsymbol{i}}(\boldsymbol{j})$ is carried by the $i$ th incoming path, and then by the $j$ th final server. 


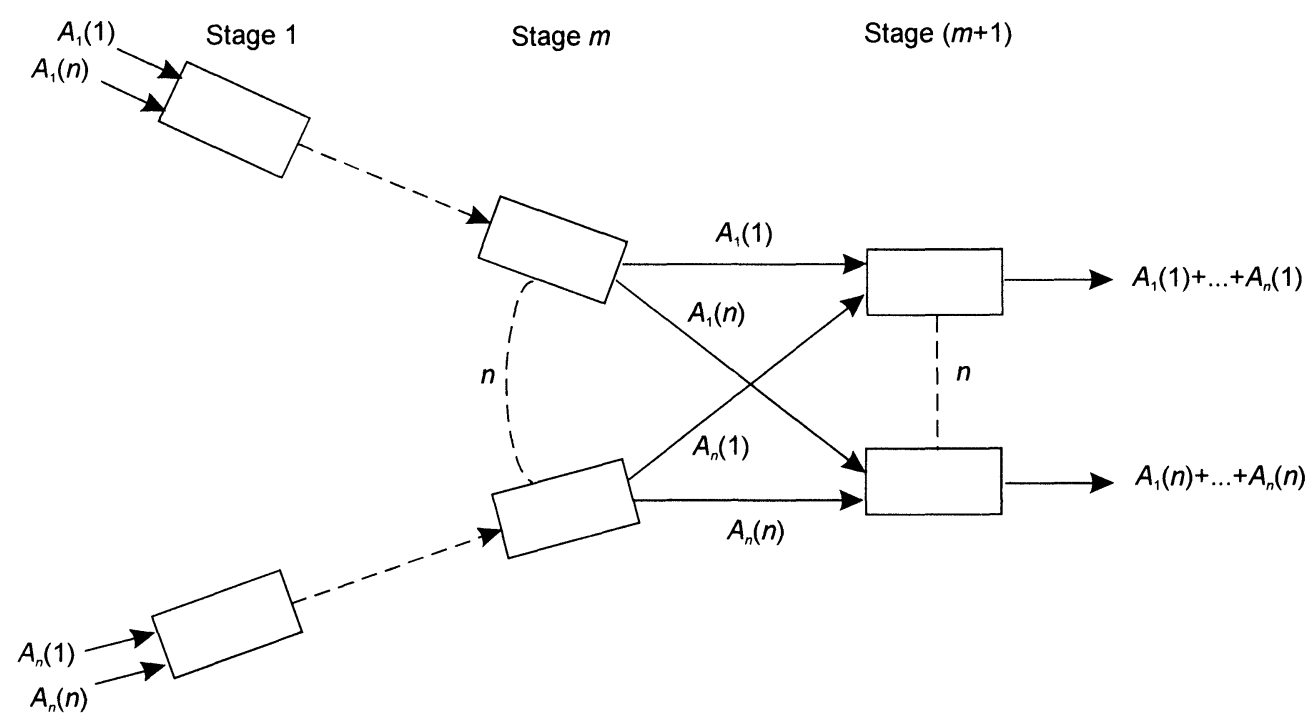

Figure 1: The Full Network

In Le Gall [1], we observe that traffic streams crossing upstream have no significant influence on the queueing delay at the final stage (due to our assumption that the busy periods are not broken up for $\boldsymbol{m}$ large), an important case in which a local arrival initiating a local busy period made the same upstream. We therefore may eliminate these streams. This also holds for intermediate arrivals along incoming paths, since their influence is not changed (at the final stage) by assuming that these customers arrive at the entry to the network, and correspond to the service times equal to zero in the first stages.

Our study may be simplified by introducing the truncated network shown in Figure 2. At the final stage, we consider only a single server. This server handles the traffic stream $\boldsymbol{A}_{\boldsymbol{i}}$ coming from the $i$ th incoming path $(i=1 \ldots n)$. In this incoming path, the traffic stream interferes with a traffic stream $\boldsymbol{B}_{\boldsymbol{i}}$, which is not offered to the final server. Its customers may be considered as "premature departures" affecting the final local queue, a phenomenon usually neglected. 


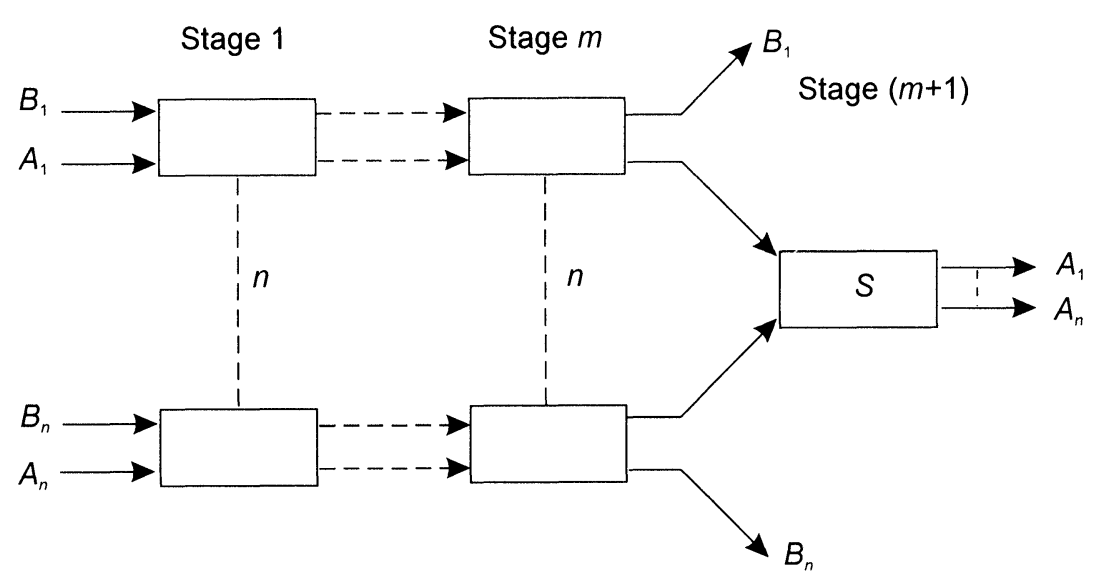

Figure 2: The Truncated Network

In the general case, we have no symmetry. The number $\boldsymbol{m}_{\boldsymbol{i}}$ of successive servers in the ith incoming path is different in each path, and successive servers may generate different service times (with different distributions). In fact, it will be possible to define a hypothetical network of Figure 2 due to some following properties.

First, we will not consider the special impact of "premature departures", related to a reduction factor (due to the concept of "apparent" upstream delays), to define an equivalent tandem queue with a number $\boldsymbol{m}$ of upstream stages (as defined by a certain relation depending on the number $\boldsymbol{m}_{\boldsymbol{i}}$ and the overall upstream service time $\boldsymbol{T}^{\prime}$ ) on the condition that the busy periods are not broken up. Moreover, if successive service times of the same customer are not too widely varying compared with the local queueing delays, this equivalent tandem queue may be assimilated to a theoretical packet switched tandem queue in which successive service times are identical. This simplifies the calculations on the condition that the number $\boldsymbol{m}$ of identical successive servers is defined correctly. In that condition, we define a virtual local queueing delay, independent of the considered incoming path. This great simplification cannot exist (for $\boldsymbol{m}$ small) when the busy periods are broken up.

Secondly, we will consider the special impact of "premature departures" (due to the concept of "apparent" upstream delays) to define the actual local queueing delay. Consequently, a reduction factor will appear to lead (in part) towards the GI/G/1 queue. This reduction factor (as a function of $B_{i}$ and the considered incoming path) may generate the same influence as a hypothetical number of identical incoming paths in Figure 2 truncated network to replace the actual number $\boldsymbol{n}$. Consequently, Figure 2 will be a reference figure in our paper.

\section{Preliminary Theory}

\subsection{The Equivalent Tandem Queue and the Virtual Local Queueing Delay}

Consider the case of a concentration tree (Figure 2) with traffic streams $A_{i}$ and $B_{i}$, with an identical traffic intensity at each stage, without taking into account a certain 
reduction factor due to the concept of "apparent" upstream delays (to be considered in Subsection 3.3). Consequently, we define a virtual local queueing delay as considered in Le Gall [1].

Each incoming path is a tandem queue, with the following notations for the $\boldsymbol{h}$ th customer at stage $k=1$...m:

- local queueing delay $w_{h}^{k}$;

- $\quad$ local service time $T_{h}^{k}$;

- $\quad$ local sojourn time $s_{h}^{k}=w_{h}^{k}+T_{h}^{k}$;

- $\quad$ interarrival interval [between customers $(h-1)$ and $h$ ] $\boldsymbol{Y}_{\boldsymbol{h}-\mathbf{1}}^{\boldsymbol{k}}$;

- $\quad$ occasional idle period [during $\left.Y_{h-1}^{k}\right] \boldsymbol{e}_{\boldsymbol{h}}^{\boldsymbol{k}}$.

In other words, we may write:

$$
Y_{h-1}^{k}=T_{h}^{k-1}+e_{h}^{k-1}
$$

Moreover, we let for $k=2 \ldots m$ :

$$
\begin{gathered}
T_{h}^{\prime}+\ldots+T_{h}^{k}=T_{h}^{1}(k) \\
s_{h}^{2}+\ldots+s_{h}^{k}=S_{h}^{k-1} .
\end{gathered}
$$

In Le Gall [2], we recall that this concentration tree (with the same traffic intensity at each stage) gives the same local queueing delay distribution (at the final stage) as does an equivalent tandem queue, concerning an arbitrary customer (coming from an arbitrary incoming path) with the same local service time $\boldsymbol{T}$, and the same upstream overall service time $\boldsymbol{T}^{\prime}$ [notation (1)]. To simplify the calculations, when the busy periods are not broken up (during the upstream busy periods), we defined an equivalent tandem queue with $\left(\boldsymbol{m}_{\mathbf{0}}+\mathbf{1}\right)$ successive identical single servers (as in packet switching), where $\boldsymbol{m}_{\mathbf{0}}$ is given by the relation:

$$
\operatorname{Var}\left(\boldsymbol{m}_{\mathbf{0}} \cdot T\right)=\operatorname{Var} \boldsymbol{T}^{\prime}
$$

if $\boldsymbol{T}$ and $\boldsymbol{T}^{\prime}$ are not constant. We deduce:

$$
\mathbf{m}_{\mathbf{0}}^{\mathbf{2}}=\frac{\operatorname{Var} T^{\prime}}{\operatorname{Var} T}
$$

When $\boldsymbol{T}$ and $\boldsymbol{T}^{\prime}$ are highly varying, we have: $\operatorname{Var}\left(m_{0} \cdot T\right) \cong m_{0}^{2} \cdot E T^{2}, \operatorname{Var} T^{\prime} \cong E \boldsymbol{T}^{\prime 2}$, and consequently,

$$
m_{0}^{2}=\frac{E T^{2}}{E T^{2}}
$$

In queueing formulae, when $\boldsymbol{m}_{\mathbf{0}}$ is between two successive integers, we will use an interpolation between delays related to these integers, or directly the possible fractional $\boldsymbol{m}_{\mathbf{0}}$ in formulae. In Le Gall [2], relation (3), and in Le Gall [3], relation (7), we gave the following condition for busy periods not broken up:

Hypothesis 1: (Busy periods not broken up) We assume that the following relation is satisfied: 


$$
T_{h}^{k-1} \leq s_{h-1}^{k}, \quad k=2 \ldots(m+1) .
$$

This is the case when:

(a) All successive service times of the same customer are identical (because in this case: $T_{h}^{k-1}=T_{h}^{k}<s_{h}^{k}=s_{h-1}^{k}$ ); and

(b) In heavy load, congestion becomes high enough to increase $s_{h-1}^{k}$.

This hypothesis is not very restrictive. To simplify in the sequel, we shall replace $\boldsymbol{m}_{\mathbf{0}}$ by $\boldsymbol{m}$. In that case, we recall in Le Gall [2] that we get th following relation at stage $(m+1)$ :

$$
\left(T_{h}^{1}+w_{h}^{2}\right)+\ldots+\left(T_{h}^{m}+w_{h}^{m+1}\right)=\operatorname{Max}\left[T_{h}^{\prime}(m), S_{h-1}^{m}-e_{h}^{1}\right]
$$

The left-hand side may be written:

$$
S_{h}^{m}+\left(T_{h}^{1}-T_{h}^{m+1}\right)
$$

To simplify calculations, we introduce a second hypothesis (see Le Gall [2]), which is usually satisfied:

Hypothesis 2: (Limitation to successive service time variations) If $\varepsilon$ is an arbitrary small positive number, we suppose that the following condition

$$
\operatorname{Lim}_{m \rightarrow \infty} \operatorname{Prob}\left(\left|\frac{T_{h}^{1}-T_{h}^{m+1}}{T_{h}^{2}+\ldots+T_{h}^{m+1}}\right|<\varepsilon\right) \rightarrow 1
$$

is satisfied for any $\boldsymbol{h}>0$.

In that case, recurrence relation (12) is equivalent (in probability for $\boldsymbol{m}$ large enough) to the stochastic recurrence [notation (7)]:

$$
S_{h}^{m}=\operatorname{Max}\left[T_{h}^{\prime}(m), S_{h-1}^{m}-e_{h}^{1}\right]
$$

Note that this equivalence could be also valid (for $\boldsymbol{m}$ large) in the case of mutual independence for successive service times, if $T_{h}^{k}(k=2, \ldots, m+1)$ is replaced by $s_{h}^{k}$ in the denominator of (13), at heave load ( $s_{h}^{k}$ being high).

Finally, Hypotheses 1 and 2 allow handling of the (almost) general case by the simple packet switched case. In Le Gall [5], we gave the virtual local sojourn time distribution (at the final stage). We let, from notations (1) and (4)

$$
\begin{gathered}
\boldsymbol{v}_{\mathbf{0}}(\boldsymbol{t})=\frac{Q_{1}}{Q_{1}(t)} \cdot F_{1}(t), \boldsymbol{v}(\boldsymbol{t}, \boldsymbol{u})=\operatorname{Exp}\left\{-u \cdot \int_{t}^{\infty} \frac{1-F_{1}(v)}{Q_{1}(v)} \cdot d v\right\} \\
\boldsymbol{u}_{\boldsymbol{m}}(\boldsymbol{t}, \boldsymbol{u})=v_{0}(t) \cdot[v(t, u)]^{m} \\
\boldsymbol{d}_{\boldsymbol{m}}(\boldsymbol{t}, \boldsymbol{u})=\int_{0}^{t} v\left(\frac{t+w}{m}, u\right) \cdot d_{w_{m-1}} u_{m}\left(\frac{w}{m-1}, u\right) .
\end{gathered}
$$


Finally, a distribution function of the virtual local sojourn time $\boldsymbol{U}(\boldsymbol{m})$, at final stage $(m+1)$, and for an arbitrary customer, is from notations (2) and (3):

(a) Case of renewal input:

$$
\boldsymbol{U}(\boldsymbol{t}, \boldsymbol{m})=\frac{1}{2 \pi i} \cdot \int_{+0} \frac{\varphi_{0}(-u) \cdot \Phi_{1}(u)}{Q_{1}} \cdot d_{m}(t, u) \cdot \frac{d u}{u}
$$

(b)

Case of Poisson input:

$$
\boldsymbol{U}(\boldsymbol{t}, \boldsymbol{m})=d_{m}(t, \lambda) \cong v_{0}(t) \cdot\left[v\left(\frac{t}{m}, \lambda\right)\right]^{m}
$$

In Le Gall [5], we also gave an approximated expression for relation (17), for the situation when $\boldsymbol{m}$ increases. Consider the longest service time $\boldsymbol{T}_{\boldsymbol{N}}$, and the service times almost so long (total arrival rate: $\lambda_{\boldsymbol{N}}$; total load: $\left.\boldsymbol{\rho}_{\boldsymbol{N}}\right)$. Since $\left(\lambda_{N} / \lambda\right)$ is low, the approximation for $\boldsymbol{U}(\boldsymbol{t}, \boldsymbol{m})$, independent of any segmentation of service time with medium lengths, is:

for $0<t \leq T_{N}$ :

$$
\boldsymbol{U}_{\mathbf{1}}(\boldsymbol{t}, \boldsymbol{m})=\left(1-\frac{\lambda_{N}}{\lambda}\right) \cdot \frac{1-\rho}{1-\left(\rho-\rho_{N}\right)} \cdot \exp \left\{\frac{-m \rho_{N}}{1-\left(\rho-\rho_{N}\right)} \cdot\left(1-\frac{t}{m \cdot T_{N}}\right)\right\}
$$

for $t>T_{N}$

$$
U_{1}(t, m)=1
$$

The impact of the longest service times comes from the "agglutination phenomen$o n$ ". During any busy period, relation (14) gives, when $e_{h}^{1}=0: S_{h}^{m}=S_{h-1}^{m}=\ldots=$ $S_{h}^{m}$, where $\boldsymbol{h}_{\mathbf{0}}$ corresponds to the customer initiating the busy period. The sojourn time is the same for any customer of the same busy period. A busy period initiated by a long service time, leading to long sojourn times inside this busy period, tends to amalgamate with subsequent busy periods. From stage to stage, the phenomenon is amplified, leading to a strong impact of the longest service times. This agglutination phenomenon leads to a local sojourn time independent of the considered incoming path. This also follows for the virtual local queueing delay.

Note: In the case of infinite support for the service time distribution function $\boldsymbol{F}_{1}(\boldsymbol{t})$, we have seen in Le Gall [1], Subsection 3.3, that a stationary condition exists when $\left[1-F_{1}(t)\right]$ decreases asymptotically as a negative exponential distribution. This is not the case for a Pareto distribution (corresponding to "Fractal' processes), which decreases asymptotically as $(a t)^{-\alpha},(\alpha>2)$. Consequently, it will be the same in a queueing network, in which a "Pareto" distribution cannot be handled (on the contrary of a single GI/G/1 server). Practically, we will restrict this paper to the case of finite support, in which $\boldsymbol{T}_{\boldsymbol{N}}$ is the longest service time.

\subsection{The Jitter Effect}

The equivalent tandem queue keeps the same order of arrivals at the entry to the network, and at final stage $(m+1)$. The actual arrival process at this final stage is different, since the incoming traffic streams are mutually independent. This difference generates a local jitter effect. independent of the above queueing delay, which was evaluated approximately in Le Gall [2], Subsection 3.1, for large $\boldsymbol{n}(>5)$. The 
distribution function of this local jitter delay is:

for $0<t \leq T^{\prime \prime}-T$ :

$$
J(t)=\frac{1-\rho^{\prime \prime}}{1-\rho^{\prime \prime} \cdot \frac{t+T}{T^{\prime \prime}}}, \text { with } T^{\prime \prime}=\frac{T}{1-\rho^{\prime \prime}}, \rho^{\prime \prime}=\left(1-\frac{1}{n}\right) \cdot \rho .
$$

for $t>T^{\prime \prime}-T$ :

$$
J(t)=1
$$

The accuracy is better when the load of long service times is lower than $0.3 \rho$.

This jitter effect is only significant for heavily loaded networks.

\subsection{The Impact of Premature Departures and the Actual Local Queueing Delay}

We now consider the special impact of "premature departures" at the final stage $(m+1)$ to define the actual local queueing delay. Without considering this special impact, and excluding the jitter effect, the virtual local sojourn time at stage $(m+1)$, as perceived at the entry to the network, is:

$$
\boldsymbol{U}(\boldsymbol{m})=\left(W_{0}+T\right)+\boldsymbol{S}(\boldsymbol{m})
$$

where $\boldsymbol{S}(\boldsymbol{m})$ is due to the $\boldsymbol{m}$ supplementary (upstream) stages. In Le Gall [2, 3], we noted that the overall delays observed upstream are defined without distinguishing customers in the upstream busy periods. In the case of "premature departures", the final queue only perceives apparent delays. In the ith incoming path (at the upstream stage), we introduce:

- total load $\boldsymbol{a}_{\boldsymbol{i}}$ (excluding the load of cross-traffic streams);

- part of this total load offered to the final queue $\boldsymbol{a}_{\boldsymbol{i}}^{\prime}$.

For an arbitrary customer (coming from the ith and from any incoming path, respectively), we introduce the following ratios (defining the numbers $\boldsymbol{n}_{\boldsymbol{i}}^{\prime}$ and $\boldsymbol{n}_{\boldsymbol{1}}$, respectively), replacing the number $\boldsymbol{n}$ in Figure 2:

$$
\frac{1}{n_{i}^{\prime}}=\frac{a_{i}^{\prime}}{a_{i}} ; \quad \frac{1}{n_{1}}=\sum_{i} \frac{a_{i}^{\prime}}{\boldsymbol{a}} \cdot\left(\frac{a_{i}^{\prime}}{a_{i}}\right), \text { with } \boldsymbol{a}=\sum_{i} a_{i}^{\prime} .
$$

Finally, from the ith incoming path, the final queue perceives the apparent delay $\boldsymbol{h}_{\boldsymbol{i}} \cdot \boldsymbol{S}(\boldsymbol{m})$, where $\boldsymbol{h}_{\boldsymbol{i}}$ is a random number $=1$, with probability $\left(\frac{1}{\boldsymbol{n}_{\boldsymbol{i}}^{\prime}}\right)$, and $=0$ with probability $\left(1-\frac{1}{\boldsymbol{n}_{\boldsymbol{i}}^{\prime}}\right)$. Stochastic expression (20) becomes, for the actual local sojourn time $\boldsymbol{S}_{\boldsymbol{i}}$ including the jitter delay $J$, with $D(m)=U(m)+J$ :

$$
\begin{gathered}
\boldsymbol{S}_{\boldsymbol{i}}=h_{i} \cdot \boldsymbol{D}(\boldsymbol{m})+\left(1-h_{\boldsymbol{i}}\right) \cdot\left(\boldsymbol{W}_{\mathbf{0}}+\boldsymbol{T}\right) \\
E e^{-z \boldsymbol{S}_{\boldsymbol{i}}}=\left(\frac{1}{\boldsymbol{n}_{\boldsymbol{i}}^{\prime}}\right) \cdot E e^{-z \boldsymbol{D}(\boldsymbol{m})}+\left(1-\frac{1}{\boldsymbol{n}_{\boldsymbol{i}}^{\prime}}\right) \cdot E e^{-z\left(\boldsymbol{W}_{\mathbf{0}}+\boldsymbol{T}\right)}
\end{gathered}
$$

Finally, in Le Gall [2, 3], we gave the following expression defining the actual local 
sojourn time $\boldsymbol{S}$ at final stage $(m+1)$, for $\operatorname{Re}(z) \geq 0$, and from any incoming path:

$$
E e^{-z S}=\left(\frac{1}{n_{1}}\right) \cdot E e^{-z D(m)}+\left(1-\frac{1}{n_{1}}\right) \cdot E e^{-z\left(\boldsymbol{W}_{0}+T\right)},
$$

with

and

$$
\boldsymbol{D}(\boldsymbol{m})=U(m)+J
$$

$$
\boldsymbol{W}_{\mathbf{0}}=\text { queueing delay of the GI/G/1 queue; } \boldsymbol{J}=\text { Jitter delay, }
$$

$\boldsymbol{U}(\boldsymbol{m})=$ virtual local sojourn time of the equivalent tandem queue (identical for each customer of the same busy period).

Conclusions:

(a) In case of a heavily loaded environment $\left(a_{i}^{\prime} \cong 0 \rightarrow\left(\frac{1}{n_{1}}\right) \cong 0\right)$, (i.e., in case of traffic streams diverging, and consequently generating a lot of "premature departures"), we have for the actual local queueing delay $\boldsymbol{w}: E e^{-z \boldsymbol{w}} \cong E e^{-z \boldsymbol{W}_{\mathbf{0}}}$. This is a classical result.

(b) In case of a slightly loaded environment $\left(a_{i}^{\prime} \cong a_{i} \rightarrow\left(\frac{1}{n_{1}}\right) \cong 1\right)$, (i.e., in case of no "premature departures"), the tandem queue effect appears with the agglutination phenomenon, increasing buffer overloads, but the value of $U(m)$ is different since the upstream traffic intensities become lower.

(c) In the general case (in stationary regime), and due to the concept of apparent queueing delay used above, we can state the basic following properties:

( $\alpha$ ) An arbitrary local customer can see the tandem queue effect (with the agglutination phenomenon and the buffer overload) with a probability $\left(1 / n_{1}\right)$, and the classical $G I / G / 1$ queue in the other cases. This probability corresponds to the case of successive local customers coming from the same incoming path.

$(\beta)$ Despite the breaking up of the traffic streams at each stage, the aggregation of small parts of upstream busy periods gives rise to a new local busy period corresponding to the maximum upstream sojourn times (see our comment on the agglutination phenomenon at the end of Subsection 3.1). Consequently, the agglutination phenomenon is amplified from stage to stage. In fact, inside a local busy period, any customer appears to be indistinguishable, and it follows that the concentration tree may be assimilated to a single equivalent tandem queue. This explains why the tandem queue effect increases from stage to stage, for a given probability $\left(1 / \boldsymbol{n}_{1}\right)$ to perceive this effect.

Note: This tandem queue effect is generated by the equivalent tandem queue, which has to satisfy Hypothesis 1 (busy periods not broke up) and 2 (limitation to successive service time variations). Consequently, the upstream loads considered are equal to the final local load, since successive equivalent servers are identical to the final server, in case of a normally loaded environment.

(d) When we may approximately use Hypothesis 2, in the case of mutual 
independent successive service times, the tandem queue effect exists for $m$ large, since the upstream service time (in case of congestion) is also the downstream interarrival time (for two successive arrivals from the same incoming path). This property is not consistent with Jackson's theory (due to the indistinguishability of customers inside any local busy period).

(e) Expression (22) proves the need to monitor the partial traffic streams loads, and not just the server load (i.e., traffic intensity). This is the same for traffic simulation methods. A direct observation of the local queueing delay cannot detect the possible correlation of the local interarrival time with the upstream service time (i.e, tandem queue effect). Finally, a classical GI/G/1 queue may be observed instead of the tandem queue effect. To avoid this difficulty, it is necessary to directly observe the difference between the two successive (upstream and local) overall sojourn times for a given traffic stream.

After having outlined our earlier papers, we now can define and evaluate the local queueing delay in a single server queueing network with varying service times, as well as for a renewal input (at the entry to the network) related to the local queueing process.

\section{The Local Queue in the Network}

We will evaluate the local queue distribution, the buffer overload, and the rejection rate in the local buffer.

\subsection{The Local Queue Distribution}

The actual local sojourn time $\boldsymbol{S}$ is defined by expression (23), referring to the classical queueing delay $\boldsymbol{W}_{\mathbf{0}}$ of the local GI/G/1 queue, and to the virtual local sojourn time $\boldsymbol{D}(\boldsymbol{m})$, sum of the jitter delayJ and the virtual local sojourn time $\boldsymbol{U}(\boldsymbol{m})$ of the equivalent tandem queue [ $\boldsymbol{J}$ and $\boldsymbol{U}(\boldsymbol{m})$ being mutually independent]. The distribution of $\boldsymbol{U}(\boldsymbol{m})$ is defined by expressions (15) and (16). When $\boldsymbol{m}$ increases, we may simplify the expression $\boldsymbol{d}_{\boldsymbol{m}}(\boldsymbol{t}, \boldsymbol{u})$, by introducing the following approximations:

$$
\boldsymbol{v}\left(\frac{t+w}{m}, u\right) \cong \boldsymbol{v}\left(\frac{t}{m}, u\right) ; \quad \boldsymbol{u}_{\boldsymbol{m}-\mathbf{1}}\left(\frac{w}{m-1}, u\right) \cong \boldsymbol{u}_{\boldsymbol{m}-\mathbf{1}}\left(\frac{w}{m}, u\right) .
$$

For $v_{\mathbf{0}}(t)$ relating to the case $m=1$ only, we may write, finally from expression (16):

$$
\boldsymbol{d}_{\boldsymbol{m}}(\boldsymbol{t}, \boldsymbol{u}) \cong \boldsymbol{v}_{\mathbf{0}}(t) \cdot\left[\boldsymbol{v}\left(\frac{t}{m}, u\right)\right]^{m} .
$$

In the case of Poisson arrivals, we have only one pole [for $\operatorname{Re}(u)>0$ ] in the integrand of expression (16): $u=\lambda$. Expression (25) above leads to the approximations in expressions (17) and (18).

\subsection{The Approximated Expression of the Distribution}

Expressions (1) define the data, related to an arbitrary local customer, defining the local GI/G/1 queue. As for expression (18), we suppose that $\boldsymbol{T}_{\boldsymbol{N}}$ corresponds to the longest service times (in case of finite support), and we define the total arrival rate 
$\lambda_{N}$ (and the total load: $\rho_{N}$ ) for the local arbitrary customers corresponding to a service time closed to $\boldsymbol{T}_{\boldsymbol{N}}$. We want to evaluate expression (25) for $\boldsymbol{t}$ closed to $\boldsymbol{T}_{\boldsymbol{N}}$. We introduce $\varphi_{1}^{*}(z)$ and $\boldsymbol{Q}_{1}^{*}$, when the customers corresponding to the $\operatorname{set}\left(\boldsymbol{\lambda}_{\boldsymbol{N}}, \boldsymbol{T}_{\boldsymbol{N}}\right)$ are excluded. From expression (4), we have:

$$
\begin{gathered}
F_{1}(t)=\frac{\sum_{j=1}^{N-1} \lambda_{j}}{\sum_{j=1}^{N} \lambda_{j}}=1-\frac{\lambda_{N}}{\lambda}, \\
\varphi_{1}(z, t)=\frac{\sum_{j=1}^{N-1} \lambda_{j}}{\sum_{j=1}^{N} \lambda_{j}} \cdot \varphi_{1}^{*}(z)=\left(1-\frac{\lambda_{N}}{\lambda}\right) \cdot \varphi_{1}^{*}(z) .
\end{gathered}
$$

Usually, $\boldsymbol{T}_{\boldsymbol{N}}$ is much longer than $\boldsymbol{E}(\boldsymbol{T})$. Consequently, $\left(\lambda_{N} / \lambda\right)$ may be neglected, and we may write:

$$
\varphi_{1}(z, t) \cong \varphi_{1}^{*}(z), \quad Q_{1}(t) \cong Q_{1}^{*}
$$

Finally, expression (25) becomes:

$$
\boldsymbol{d}_{\boldsymbol{m}}(\boldsymbol{t}, \boldsymbol{u}) \cong\left(1-\frac{\lambda_{N}}{\lambda}\right) \cdot \frac{Q_{1}}{Q_{1}^{*}} \cdot \exp \left\{-\frac{u}{\lambda} \cdot \frac{m \cdot \rho_{N}}{Q_{1}^{*}} \cdot\left[1-\frac{t}{m \cdot T_{N}}\right]\right\}
$$

For $u=\lambda$, in the case of Poisson arrivals, expression (28) leads to approximation (18) and, in Le Gall [5], we have seen that this approximation (18) is practically valid for any time $t$. Consequently, expressions (16) and (28) are useful approximations to evaluate the distribution of the virtual local sojourn time $U(m)$ for any time $t$.

\subsection{The Buffer Load}

First, we want to evaluate the mean $\overline{U(m)}$. We let:

$$
\boldsymbol{A}(u)=\frac{\rho_{N}}{Q_{1}^{*}} \cdot \frac{u}{\lambda}
$$

In expression (16), if we replace $d_{m}(t, u)$ by one, $U(t, m)$ becomes equal to one. Consequently,

We let:

$$
1-U(t, m)=\frac{1}{2 \pi i} \cdot \int_{+0} \frac{\varphi_{0}(-u) \cdot \Phi_{1}(u)}{Q_{1}} \cdot\left[1-d_{m}(t, u)\right] \cdot \frac{d u}{u} .
$$

$$
\overline{U(m)}=\int_{0}^{T_{N}}[1-U(t, m)] \cdot d t
$$




$$
D(m, u)=\int_{0}^{T_{N}}\left[1-d_{m}(t, u)\right] \cdot d t
$$

From expressions (16), (28) and (29), we deduce:

with

$$
\overline{\boldsymbol{U}(\boldsymbol{m})}=\frac{1}{2 \pi i} \cdot \int_{+0} \frac{\varphi(-u) \cdot \Phi_{1}(u)}{Q_{1}} \cdot \boldsymbol{D}_{\boldsymbol{m}}(\boldsymbol{u}) \cdot \frac{d u}{u},
$$

$$
\boldsymbol{D}_{\boldsymbol{m}}(\boldsymbol{u})=\boldsymbol{T}_{\boldsymbol{N}} \cdot\left\{1-\left(1-\frac{\lambda_{N}}{\lambda}\right) \cdot \frac{Q_{1}}{Q_{1}^{*}} \cdot \frac{\operatorname{Exp}[-(m-1) \cdot A(u)]-\operatorname{Exp}[-m \cdot A(u)]}{A(u)}\right\} .
$$

Finally, taking definitions (1) and relations (23) into account, we deduce the mean actual local sojourn time [at stage $(m+1)$ ] of an arbitrary customer, corresponding to his occupancy in the local buffer:

$$
\boldsymbol{s}_{\mathbf{1}}(\boldsymbol{m}+\mathbf{1})=\frac{1}{n_{1}} \cdot[\overline{U(m)}+\bar{J}]+\left[1-\frac{1}{n_{1}}\right] \cdot\left[\overline{W_{0}}+\bar{T}\right]
$$

with $\overline{\boldsymbol{U}(\boldsymbol{m})}$ being given by expression (32). In the same way, we could evaluate the second moment.

\subsection{The Buffer Rejection Rate}

If $\boldsymbol{K}$ is the buffer capacity, a customer is rejected on his local arrival if the number of customers $j$ waiting is such as: $j \geq K-1$. If $j$ denotes the number of customers in the local system (waiting or with service in progress), the rejection condition becomes: $j \geq K$. We suppose that a rejected customer repeats his arrival at the entry to the network. It follows that traffic handled locally is not decreasing, with the queue length distribution giving a good approximation of the rejection rate, when its value is low.

In conclusion $(c)$ of Subsection 3.3, we noted that an arbitrary local customer can see the tandem queue effect (with its rejection rate) with a probability $\left(1 / \boldsymbol{n}_{\mathbf{1}}\right)$, including the jitter effect, and the classical GI/G/1 queue (with its rejection rate) in the other cases. We introduce the following notation for an arbitrary local customer (in stationary regime):

- $\quad \boldsymbol{R}_{\mathbf{0}}(\boldsymbol{K})$ rejection rate due to the $\mathrm{GI} / \mathrm{G} / 1$ queue;

- $\boldsymbol{R}_{\mathbf{1}}(\boldsymbol{K})$ rejection rate due to the tandem queue;

- $\quad \boldsymbol{R}(\boldsymbol{K})$ the total rejection rate.

Note that the rejection rate due to the jitter effect may be neglected. Due to our comment above, and using relation (23), we may write:

$$
\boldsymbol{R}(\boldsymbol{K})=\left(\frac{1}{n_{1}}\right) \cdot R_{1}(K)+\left(1-\frac{1}{n_{1}}\right) \cdot R_{0}(K) .
$$

(a) Evaluation of $\boldsymbol{R}_{\mathbf{0}}(\boldsymbol{K})$. For the classical, local GI/G/1 queue, we recall our notation (2). $\quad \boldsymbol{F}_{\mathbf{0}}(\boldsymbol{t})$ is the distribution function of the interarrival intervals (at the entry to the network). $W_{0}(t)$ is the distribution function of the local queueing delay. Expression (35) in Le Gall [4] gives: 


$$
\boldsymbol{R}_{\mathbf{0}}(\boldsymbol{K})=\int_{0}^{\infty}\left[1-W_{0}(t)\right] \cdot d\left[F_{0}(t)\right]^{(K-1)}
$$

where $\left[\boldsymbol{F}_{\mathbf{0}}(\boldsymbol{t})\right]^{(K)}$ denotes the $\boldsymbol{K}$-fold convolution of $F_{0}(t)$.

(b) Evaluation of $\boldsymbol{R}_{\mathbf{1}}(\boldsymbol{K})$. Following our comment after expression (18), related to the agglutination phenomenon of short service times behind a long service time initiating a local busy period, the interarrival times $\boldsymbol{T}^{*}$, during this busy period, correspond to the service times (excluding the longest service times $\left.\boldsymbol{T}_{\boldsymbol{N}}\right)$. Due to notations $(26)$ and $(27)$, we denote by $\boldsymbol{F}_{\mathbf{1}}^{*}(\boldsymbol{t})$ the service time distribution function, excluding the set $\left(\lambda_{N}, T_{N}\right)$. From notation (6), the rejection condition, at stage $(m+1)$, becomes:

$$
\begin{gathered}
s_{h}^{m+1}-\left(Y_{h}^{m+1}+\ldots+Y_{h+K-1}^{m+1}\right)>0, \\
\left(\text { i.e., } s_{h}^{m+1}-\boldsymbol{K} \cdot \boldsymbol{T}^{*}>0\right),
\end{gathered}
$$

which leads to expression:

$$
\begin{aligned}
& \boldsymbol{R}_{\mathbf{1}}(K)=\int_{0}^{T_{N}}[1-U(t, m)] \cdot d\left[F_{1}^{*}(t)\right]^{K}, \text { if } K \leq \frac{T_{N}}{T_{1}} \\
&=0, \text { if } K>\frac{T_{N}}{T_{1}}
\end{aligned}
$$

where $\left[F_{1}^{*}(t)\right]^{K}$ denotes the $K$-fold convolution of $\boldsymbol{F}_{\mathbf{1}}^{*}(\boldsymbol{t}) \cdot \boldsymbol{T}_{\mathbf{1}}$ corresponds to the shortest service times. $\boldsymbol{U}(\boldsymbol{t}, \boldsymbol{m})$ is defined by expressions (16) and (15), or more simply by approximation (25). In case of Poisson input, $\boldsymbol{U}(\boldsymbol{t}, \boldsymbol{m})$ is defined by simple expression (17).

\subsection{Conclusion}

Due to expressions (32) and (37), introducing a limitation to $\left(T_{N} / T_{1}\right)$ for the impacts of tandem queue load and length, respectively, an important property follows from relation (23), from combining the tandem queue and GI/G/1 queue models:

- "For a queue length longer and a buffer capacity larger than $\left(T_{N} / T_{1}\right)$, the $G / G / 1$ queue model alone exists."

If this property, typical for single server queueing networks with varying service times (without breaking up the busy periods), is not perceived, it may be a real danger to use only the GI/G/1 queue model for the design, dimensioning, and management of the network, due to significant buffer overloads not being perceived. Buffer congestion leads to servers' inaccessibility, and the agglutination phenomenon is transmitted downstream (and upstream by reattempts) immediately, generating the blocking of a large area in the network.

The usual concept of local traffic source should be revised in some network queueing theories (e.g., product form theory), due to the existence of the underlying tandem queue effect. In Markovian queueing networks, particularly, the concept of a local transition coefficient is not consistent with the tandem queue model above. And finally, some standards could be very useful for introducing a limitation to the ratio $\left(T_{N} / T_{1}\right)$, a typical constraint when service times are varying and not perceived up to now. 


\section{Case of a Packet Switch Network}

\subsection{The Packet Traffic Streams}

The truncated network of Figure 2 is our reference figure, with traffic streams $\boldsymbol{A}_{\boldsymbol{i}}$ and $\boldsymbol{B}_{\boldsymbol{i}}$. Even though traffic streams are not identical, to define the equivalent tandem queue (of Subsection 3.1) with the same local queue at the final stage (for an arbitrary packet), we may consider identical traffic streams in each incoming path.

The total traffic stream (at final stage) handles a mixture of $\boldsymbol{N}$ packet populations, each labeled $j(j=1 \ldots N)$. Each population corresponds to a partial Poisson traffic stream $j$ with constant (i.e., deterministic) packet length $T_{j}\left(T_{1}<T_{2}\right.$ $<\ldots<T_{N}$ ), a partial arrival rate $\boldsymbol{\lambda}_{\boldsymbol{j}}$, and a partial load (in stationary regime) $\boldsymbol{\rho}_{\boldsymbol{j}}=$ $\lambda_{j} \cdot T_{j}$. For the total traffic stream, the total arrival rate (for an arbitrary packet) is: $\lambda=\sum_{j=1}^{N} \lambda_{j}$, and the total load is: $\rho=\sum_{j=1}^{N} \rho_{j}$. With notation (2), we compute for the transform of the sending (i.e., service) time distribution function of any packet, and for $\operatorname{Re}(z) \geq 0$ :

$$
\varphi_{1}(z)=\sum_{j=1}^{N} \frac{\lambda_{j}}{\lambda} \cdot e^{-z T} .^{-z}
$$

\subsection{The Distribution of the Local Queue}

Relation (23) defines the distribution of the actual local sojourn time $\boldsymbol{S}$ with two components:

- the case of the M/G/1 queue (well known);

- the case of the equivalent tandem queue.

The distribution function $\boldsymbol{U}(\boldsymbol{t}, \boldsymbol{m})$ of the virtual local sojourn time $\boldsymbol{U}(\boldsymbol{m})$, at the final stage of the equivalent tandem queue, as defined by expressions (15) and (17), has been given in Le Gall [5], formula (36):

for $t<T_{1}$ :

for $T_{k}<t<T_{k+1}$ :

$$
U_{0}(t, m)=0
$$

with

$$
\begin{gathered}
v_{0}(t)=\frac{\lambda_{1}+\ldots+\lambda_{k}}{\lambda} \cdot \frac{1-\rho}{1-\left(\rho_{1}+\ldots+\rho_{k}\right)} ; \\
v(t, \lambda)=\operatorname{Exp}\left\{-\left(\frac{\lambda_{k+1}+\ldots+\lambda_{N}}{1-\left(\rho_{1}+\ldots+\rho_{k}\right)} \cdot\left(T_{k+1}-t\right)+\ldots\right.\right. \\
\left.\left.+\frac{\lambda_{N}}{1-\left(\rho_{1}+\ldots+\rho_{N-1}\right)} \cdot\left(T_{N}-T_{N-1}\right)\right)\right\}
\end{gathered}
$$

for $t>T_{N}$ :

$$
U_{N}(t, m)=1 .
$$


In fact, when $\boldsymbol{m}$ increases, a good approximation $U_{\mathbf{1}}(\boldsymbol{t}, \boldsymbol{m})$ is given by expression (18), depending only on the set $\left(\lambda_{N}, T_{N}\right)$.

\subsection{The Buffer Load}

The mean actual local sojourn time [at stage $(m+1)$ ] of an arbitrary packet (from any incoming path), corresponding to its occupancy in the local buffer, may be written from definitions (1) and relation (23):

$$
\overline{\boldsymbol{S}}=s(m+1)=\frac{1}{\boldsymbol{n}_{\mathbf{1}}} \cdot[\overline{U(m)}+\bar{J}]+\left[1-\frac{1}{\boldsymbol{n}_{\mathbf{1}}}\right] \cdot\left[\overline{W_{0}}+\bar{T}\right]
$$

In expression (33), $\overline{\boldsymbol{U}(\boldsymbol{m})}$ is given by approximated expression (32), but here $\overline{\boldsymbol{U}(\boldsymbol{m})}$ has to be deduced from expressions (39).

(a) Exact expression $\boldsymbol{s}(\boldsymbol{m}+1):$

( $\alpha)$ Calculation of $\overline{\boldsymbol{U}(\boldsymbol{m})}$

From expressions (39), we may write:

$$
\overline{\boldsymbol{U}(\boldsymbol{m})}=\sum_{k=0}^{N-1} \int_{T_{k}}^{T_{K}+1}\left[1-U_{k}(t, m)\right] \cdot d t
$$

( $\beta$ ) Calculation of $\overline{\boldsymbol{J}}$

In Figure 2, we have $\boldsymbol{n}$ incoming paths. Expression (19) gives:

$$
\bar{J}=\int_{0}^{T^{\prime \prime}-T}[1-J(t)] \cdot d t
$$

( $\gamma) \quad$ Calculation of $\left(\overline{\boldsymbol{W}_{\mathbf{0}}}+\overline{\boldsymbol{T}}\right)$

From expression (38), we deduce that for the service time distribution of an arbitrary packet:

$$
\bar{T}=E(T)=\sum_{j=1}^{N} \frac{\lambda_{j}}{\lambda} \cdot T_{j}, m_{2}=E\left(T^{2}\right)=\sum_{j=1}^{N} \frac{\lambda_{j}}{\lambda} \cdot\left(T_{j}\right)^{2}
$$

The classical Pollaczek formula gives:

$$
\overline{W_{0}}=\frac{1}{2} \cdot \frac{\rho}{1-\rho} \cdot \frac{m_{2}}{T} .
$$

Depending on the actual incoming paths, expression (21) defines $\boldsymbol{n}_{\mathbf{1}}$ and, finally, we may evaluate the buffer occupancy $s(\boldsymbol{m}+1)$, at stage $(m+1)$, as defined by expression $(40)$.

(b) Approximated expression $s_{1}(m+1)$ : A general approximated expression $\boldsymbol{s}_{\mathbf{1}}(\boldsymbol{m}+1)$ of the buffer occupancy (for an arbitrary packet) is given by (33), using (32) for $u=\lambda$ in case of Poisson arrivals, with $Q_{1}^{*}=\left(1-\left(\rho-\rho_{N}\right)\right.$ :

$$
\overline{\boldsymbol{U}(\boldsymbol{m})}=D_{\boldsymbol{m}}(\lambda)=T_{N} \cdot\left\{1-\left(1-\frac{\lambda_{N}}{\lambda}\right) \cdot \frac{1-\rho}{1-\left(\rho-\rho_{N}\right)} \cdot \frac{\operatorname{Exp}[-(m-1) A]-\operatorname{Exp}[-m A]}{A}\right\}
$$


with

$$
A=\frac{\rho_{N}}{1-\left(\rho-\rho_{N}\right)}
$$

Note: It follows that the virtual sojourn time distribution is not practically changed when we segment packets of medium lengths.

A Numerical Example: It may be interesting to consider the case of Figure 2, with a load $\boldsymbol{\rho}$ in each incoming path $\boldsymbol{i}(i=1 \ldots n)$, and in the considered terminal link $\boldsymbol{j}$. But we introduce some dissymmetry in the distribution of this load by introducing the following distribution $\boldsymbol{\rho}_{\boldsymbol{i}}(\boldsymbol{j})$, related to the load of the partial traffic stream $\boldsymbol{A}_{\boldsymbol{i}}(\boldsymbol{j})$ with $\boldsymbol{j}$ being given:

$$
\rho_{j}(j)=\rho_{0}, \quad \rho_{i}(j)=\frac{\rho-\rho_{0}}{n-1} \quad(i \neq j) .
$$

We let:

$$
\rho_{0}=h \cdot \frac{\rho}{n} \quad(h=1 \ldots n)
$$

In relations (33) and (40), $\boldsymbol{n}_{\mathbf{1}}$ is defined by expression (21), which gives:

$$
\frac{1}{n_{1}}=\frac{\rho_{0}}{\rho} \cdot\left(\frac{\rho_{0}}{\rho}\right)+\frac{\rho-\rho_{0}}{\rho} \cdot\left(\frac{\rho-\rho_{0}}{n-1} \cdot \frac{1}{\rho}\right)=\left(\frac{\rho_{0}}{\rho}\right)^{2}+\frac{1}{n-1} \cdot\left(\frac{\rho-\rho_{0}}{\rho}\right)^{2} .
$$

In fact, the buffer load increases with $\rho_{\mathbf{0}}$, the load of the partial traffic stream $\boldsymbol{A}_{\boldsymbol{j}}(\boldsymbol{j})$, which is its contribution to the total load $\boldsymbol{\rho}$ of the terminal link $\boldsymbol{j}$ [at stage $(m+1)$ ]. This contribution may be much higher than the other contributions $\boldsymbol{\rho}_{\boldsymbol{i}}(\boldsymbol{j})$. The case $h=1$ is the pure symmetrical case: $\rho_{i}(j)=\rho_{j}(j)=\frac{\rho}{n}$. On the contrary, the case $h=10(=n)$ corresponds to $\boldsymbol{A}_{\boldsymbol{j}}(\boldsymbol{j})$ becoming a pure tandem queue. For the intermediate case $h=5, \rho_{0}=\frac{\rho}{2}$, half of the total load (at the final stage) comes from a single incoming path $\left(N^{0} j\right)$; the tandem queue effect begins to appear.

In Table 1, we consider the case of an "IP" (Internet Protocol) traffic: $n=10$, $m=6, \rho=0.6$, and a total packet traffic stream with three partial traffic streams:

$$
T_{1}=1, T_{2}=5, T_{3}=30 ; \text { and } \rho_{1}=\rho_{2}=\rho_{3}=0.2 \text {. }
$$

\begin{tabular}{|c|c|c|c|c|c|c|}
\cline { 3 - 7 } \multicolumn{2}{c|}{} & \multicolumn{2}{c|}{ exact } & \multicolumn{2}{c|}{ approximated } \\
\hline 1 & $n_{1}$ & $\rho_{\mathbf{0}}$ & $s(m+1)$ & $C$ & $s_{1}(m+1)$ & $C_{1}$ \\
5 & 10 & 0.06 & 13.08 & 1.14 & 13.04 & 1.14 \\
10 & 3.6 & 0.30 & 16.01 & 1.40 & 15.90 & 1.39 \\
& 1 & 0.60 & 27.91 & 2.44 & 27.52 & 2.41 \\
\hline \multicolumn{2}{|l}{ M/G/1 queue: $\overline{\boldsymbol{W}_{\mathbf{0}}}+\overline{\boldsymbol{T}}$} & 11.43 & & & \\
\hline
\end{tabular}

Table 1: Mean Actual Local Sojourn Time (= Occupancy in the Local Buffer) $\boldsymbol{s}(\boldsymbol{m}+1)$, Formula $(40)$, and Overload Coefficient $\boldsymbol{C}=\frac{s(m+1)}{\overline{W_{0}}+\bar{T}}$, in Figure 2 as a Function of the Contribution of $\boldsymbol{A}_{\boldsymbol{j}}(\boldsymbol{j})$, load $\boldsymbol{\rho}_{\mathbf{0}}$, to the Total Terminal Link's Load $\rho\left(\mathrm{N}^{\mathrm{O}} j\right)$. 
Approximated Values: $s_{1}(\boldsymbol{m}+1)$, formulae (33) and (45), and $\boldsymbol{C}_{\mathbf{1}}=\frac{s_{1}(m+1)}{\overline{W_{0}}+\bar{T}}$.

Data: $n=10, m=6, \rho=0.6, \rho_{j}(j)=\rho_{0}=h \cdot \frac{\rho}{n}$.

Traffic stream (3 components): $T_{1}=1, T_{2}=5, T_{3}=30 ; \rho_{1}=\rho_{2}=\rho_{3}=0.2$.

We give the overload coefficients:

$$
C=\frac{s(m+1)}{\overline{W_{0}}+\bar{T}} ; \quad C_{1}=\frac{s_{1}(m+1)}{\overline{W_{0}}+\bar{T}}
$$

For the usual case $(h=1)$, the buffer load is just $14 \%$ higher than the buffer load given by the M/G/1 queue model. For $h=5$, the buffer load is already $40 \%$ more higher. Moreover, following expression (22), the buffer occupancy related to the traffic stream $\boldsymbol{A}_{\boldsymbol{j}}(\boldsymbol{j})$ is even $80 \%$ higher than the buffer load given by the $\mathrm{M} / \mathrm{G} / 1$ queue model. For $h=10$, the buffer load becomes $144 \%$ higher (i.e., case of the tandem queue model). Finally, the tandem queue effect becomes significant when half of the local load comes from a single incoming path only (case $h=5$ ). An example of this is the case of a virtual circuit, or of a traffic stream concentration through a supplementary upstream node.

The approximation $\boldsymbol{s}_{\mathbf{1}}(\boldsymbol{m}+\mathbf{1})$ is a good approximation, proving that the set $\left(\lambda_{N}, T_{N}\right)$ of the longest service times is sufficient to generate the agglutination phenomenon.

\subsection{The Buffer Rejection Rate}

To evaluate the buffer rejection rate $\boldsymbol{R}(\boldsymbol{K})$ in a stationary regime, we use expression (34), which causes us to evaluate the rejection rate $\boldsymbol{R}_{\mathbf{0}}(\boldsymbol{K})$ due to the M/G/1 queue model, as well as the rejection rate $\boldsymbol{R}_{1}(\boldsymbol{K})$ due to the tandem queue model, where $\boldsymbol{K}$ is the buffer capacity.

(a) Expression of $\boldsymbol{R}_{\mathbf{0}}(\boldsymbol{K}): \boldsymbol{R}_{\mathbf{0}}(\boldsymbol{K})$ is given by expression (35) for the GI/G/1 queue model. In Le Gall [4], formula (28), the expression has been given for the $M / G / 1$ queue model:

with

$$
\boldsymbol{R}_{\mathbf{0}}(\boldsymbol{K}) \cong \int_{T_{N}}^{\infty}\left[1-W_{1}(t)\right] \cdot H(t, K-2) \cdot \lambda d t
$$

$$
H(t, j)=e^{-\lambda t} \cdot \frac{(\lambda t)^{j}}{j !}
$$

$W_{1}(t)$ corresponds to the asymptotic expression of the queueing delay distribution $\boldsymbol{W}_{\mathbf{0}}(\boldsymbol{t})$ for the M/G/1 queue model. In Le Gall [4], expression (32) gives:

$$
\boldsymbol{W}_{\mathbf{1}}(\boldsymbol{t}) \cong 1-\frac{1-\rho}{\rho_{1} \cdot e^{\beta_{0} T_{1}}+\ldots+\rho_{N} \cdot e^{\beta_{0} T_{N}}-1} \cdot e^{-\beta_{0} t}
$$

where $q=\beta_{0}(>0)$ is the real (positive) root, closest to the origin, of the equation:

$$
q+\lambda-\lambda_{1} \cdot e^{q T_{1}}-\ldots-\lambda_{N} \cdot e^{q T} N=0
$$


Expressions (50) and (52) may be used, as a useful approximation, for $\rho<0.8$ and $R_{0}(K) \leq 10^{-2}$.

(b) Expression of $\boldsymbol{R}_{\mathbf{1}}(\boldsymbol{K}): \quad \boldsymbol{R}_{\mathbf{1}}(\boldsymbol{K})$ is given by expression (37), where $\boldsymbol{U}(\boldsymbol{t}, \boldsymbol{m})$ is given by the approximated expression (18). To evaluate $\left[F_{1}^{*}(t)\right]^{K}$, we consider the Laplace transform, excluding the set $\left(\lambda_{N}, T_{N}\right)$ :

$$
\left[\varphi_{1}^{*}(z)\right]^{K}=\sum_{j=0}^{K} K ! \frac{\left(\frac{\lambda_{1}}{\lambda_{1}+\lambda_{2}}\right)^{j}}{j !} \cdot e^{-j z T_{1}} \cdot \frac{\left(\frac{\lambda_{2}}{\lambda_{1}+\lambda_{2}}\right)^{K-j}}{(K-j) !} \cdot e^{-(K-j) z T_{2}},
$$

with the condition (for a single busy period): $j T_{1}+(K-j) T_{2}<T_{3}$. Expression (37) gives:

$$
\boldsymbol{R}_{\mathbf{1}}(K)=\sum_{j=0}^{K} K ! \frac{\left(\frac{\lambda_{1}}{\lambda_{1}+\lambda_{2}}\right)^{j}}{j !} \cdot \frac{\left(\frac{\lambda_{2}}{\lambda_{1}+\lambda_{2}}\right)^{K-j}}{(K-j) !} \cdot\left[1-U\left(j T_{1}+(K-j) T_{2}, m\right)\right]
$$

with $j$ such as: $j T_{1}+(K-j) T_{2}<T_{3}$.

Practically, this last condition leads only to the case $j=K$; the rejections are generated by the shortest packets.

(c) A Numerical Example: We consider the same example as in Subsection 5.3.c and in Table 1. The expression of $\boldsymbol{n}_{1}$ is defined by expression (48). Table 2 gives the numerical results for $\boldsymbol{R}_{0}(K)=10^{-2}, 10^{-3}$ and $10^{-4}$, corresponding to the buffer capacity $K=19,28$ and 40 , respectively.

\begin{tabular}{|c|c|c|c|c|c|c|}
\cline { 4 - 6 } \multicolumn{2}{c|}{} & $K$ & 19 & 28 & 40 \\
\cline { 4 - 7 } & $R_{0}(K)$ & $10^{-2}$ & $10^{-3}$ & $10^{-4}$ \\
\hline 1 & $n_{1}$ & $\rho_{0}$ & $C(K)$ & & & \\
\hline 5 & 3.6 & 0.06 & & 3.9 & 26 & 0.90 \\
\hline 10 & 1 & 0.30 & & 8.9 & 70 & 0.72 \\
\hline
\end{tabular}

Table 2: Local Rejection Rate $\boldsymbol{R}(\boldsymbol{K})=C(K) \cdot R_{0}(K)$, for a Buffer Capacity $\boldsymbol{K}$, Formulae (34) and (54). (Examples of Table 1)

[Rejection rate in the $\mathrm{M} / \mathrm{G} / 1$ queue model: $\boldsymbol{R}_{\mathbf{0}}(\boldsymbol{K})$, formula (50)]

For $K=19$ and 28 , a strong influence of the agglutination phenomenon appears: $\quad \boldsymbol{R}(\boldsymbol{K})=C(K) \cdot R_{0}(K)$, with a high value for $C(K)$. For $K>30$, this phenomenon has disappeared: $C(K)<1$.

Due to the impact of the shortest packets, we may avoid the rejections due to the agglutination phenomenon if the buffer capacity $\boldsymbol{K}$ is such as:

$$
K>\frac{T_{N}}{T_{1}}
$$


where $\boldsymbol{T}_{\boldsymbol{N}}$ and $\boldsymbol{T}_{\mathbf{1}}$ are the longest and shortest packet lengths, respectively. And this conclusion to dimension the buffer is the same as for the M/G/1 queue alone: See Le Gall [4], Subsection 4.3. Moreover, we may note that this condition (55) does not change if we segment the medium packet lengths into the shortest packet lengths. In that case, we can say that condition (55) states that the buffer capacity should be higher than the agglutination size (after segmentation).

\section{Conclusion}

(a) We have studied the single server queueing networks (excluding ring networks), when successive service times are different (or not) without breaking up the busy periods from stage-to-stage, leading to a great approximate simplification: the existence of an equivalent tandem queue effect. At the end of Subsection 3.1, and in case of infinite support for the service time distribution function $\boldsymbol{F}_{\mathbf{1}}(\boldsymbol{t})$, we stressed the need that $\left[1-F_{1}(t)\right]$ decreases exponentially at infinity. In particular, in case of a "Pareto" distribution (for "Fractal" processes) decreasing as $(a t)^{-\alpha},(\alpha>2)$, this kind of traffic cannot be carried in queueing networks (on the contrary of a single GI/G/1 server) because of the tandem queue effect: in this case link overload generates more successive local arrivals from the same incoming path. Practically, we restricted this paper to the realistic case of finite support.

(b) Due to the possible correlation between two successive local arrivals from the same incoming path, a curious "double faced" traffic model appears, as for the Janus divinity: An agglutination phenomenon (of short service times $\boldsymbol{T}_{\mathbf{1}}$ behind a long service time $\left.\boldsymbol{T}_{\boldsymbol{N}}\right)$ results for queue lengths and buffer capacities lower than $\left(T_{N} / T_{1}\right)$, and the classical GI/G/1 queue for queue lengths and buffer capacities higher than $\left(T_{N} / T_{1}\right)$. This property (amplifying from stage-to-stage) has not yet been detected with classical network queueing theories, (e.g., Markovian queues, product form theory), due to an incorrect concept of local traffic source (i.e., eliminating the occurrence of some agglutination and the concept of upstream apparent delay). These theories assumed the local combination of distinguishable customers (with distinguishable queueing delay), instead of parts of upstream busy periods with indistinguishable customers (and indistinguishable queueing delay depending on the maximum sojourn time initiating the new downstream busy period). These theories cannot be consistent with the concept of the equivalent tandem queue.

(c) Some significant consequences appear, since any link overload comes from a given incoming path which generates the tandem queue effect (i.e., correlation between local interarrival time and upstream service time). The usual queueing standards (related to long queues) cannot protect against subsequent, significant overloads in the buffers due to some possible "agglutination phenomenon" (related to short queues). Usual network management methods should be revised, and should monitor the partial traffic streams loads (and not only the server load). Moreover, when we connect two networks (or a network and a user), as in circuit switching (with buffer associated), it follows that $n_{1}=1$ in expression (23), and the inlet (downstream) works with a sojourn time (= local transfer delay) practically equal to $T_{N}$ instead of $\left(W_{0}+T\right)$, leading to a supplementary mean transfer delay $\left[T_{N}-\left(W_{0}+T\right)\right]$. This is the same in the case of a traffic stream concentration through a supplementary upstream node. In that case, leading to some packet re- 
jections in connectionless IP-based telecommunication networks, some moving pictures on the screen may be stopped a long time before continuing.

$(d)$ This longest packet length, $T_{N}$, may be bounded in a "virtual circuit mode" (depending on the considered link). On the contrary, in a "connectionless mode", the usual routing (or flow control) methods cannot be efficient to bound $T_{N}$ for a given link with its buffer. Finally, buffer dimensioning [see condition (55)] depends on the limitation needed by the network itself. Any change on this general limitation may disturb all the buffers of the network.

This disturbance may become considerable when the network tries to handle bursty traffics with non-transparent bursts (i.e., with no possibility that other packets cross the burst inside the intervals between packets of the considered burst). If $\boldsymbol{L}$ is the maximum burst duration, $\boldsymbol{T}_{\boldsymbol{N}}$ now has to be replaced by $\boldsymbol{L}$ in condition (55), leading to a ratio $\left(L / T_{1}\right)$ much greater than $\left(T_{N} / T_{1}\right)$ and, consequently, to an agglutination phenomenon much higher in the buffers (and not detected by flow control). Due to congestion in buffers, a large area of the network may rapidly become inaccessible. The classical traffic modeling, with local Poisson arrivals and no correlation with upstream service times, could not lead to this important consequence (even when successive service times are mutually independent, due to Hypothesis 2).

(e) The traffic modeling for this case of correlated, and different successive service times is more general than the case of constant service times. The occurrence of agglutinations generates the concept of indistinguishability as in nuclear physics, which may affect the concept of a local transition coefficient in Markovian queues, and the concept of product form. Moreover, this concept of upstream indistinguishability introduces some strong interferences with "premature departures", leading to the new concepts of virtual and actual local queueing delays, respectively.

$(f)$ Finally, we stress our conclusion $(e)$ in Subsection 3.3. To be sure that the tandem queue effect may be detected by traffic simulation methods, it is necessary to directly observe the two successive (upstream and local) overall sojourn times for a local arrival in a given traffic stream, instead of directly observing the local queueing delay for an arbitrary arrival. In this latter case, a simple, local Poisson arrival process without any correlation with the upstream stage could appear, leading to the evaluation of a simple, apparent $M / G / 1$ local server. The whole observation of the network could be altered, and in case of rejected packets repeated, it could be impossible to understand some high rejection rates observed, even in the case of local and total load at a medium level (e.g., 0.5 erlang), when condition (55) is not satisfied.

\section{References}

[1] Le Gall, P., Bursty traffic in packet switched networks, Proc. ITC-14 (Antibes, France, June 1994), The Fund. Role of Teletraffic in the Evolution of Telecom. Networks, Elsevier Science B.V., 1a (1994), 535-549.

[2] Le Gall, P., The theory of networks of single server queues and the tandem queue model, J. of Appl. Math and Stoch. Anal. 10:4 (1997), 363-381.

[3] Le Gall, P., Multiserver queueing networks and the tandem queue model, J. of Appl. Math. and Stoch. Anal. 11:3 (1998), 377-390.

[4] Le Gall, P., The queue-length in GI/G/s queues, Math. Prob. in Eng. 6:1 (2000), 1-11.

[5] Le Gall, P., The stationary local sojourn time in single server tandem queues 
with renewal input, J. of Appl. Math. and Stoch. Anal. 12:4 (1999), 417-428.

[6] Pollaczek, F., Problèmes stochastiques posés par le phénomène de formation d'une queue d'attente à un guichet et par des phénomènes apparentés, Mémorial des Sciences Mathématiques, Gauthier-Villars, Paris CXXXVI (1957). ( = GI/G/1 queue; in French). 


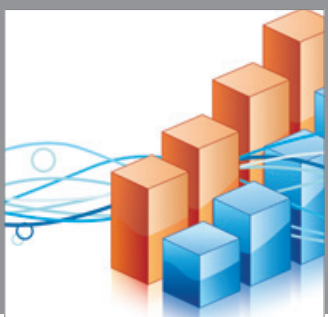

Advances in

Operations Research

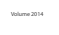

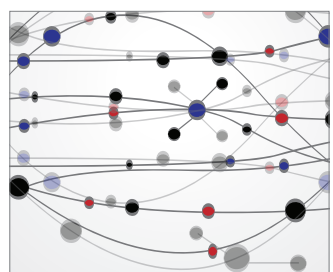

\section{The Scientific} World Journal
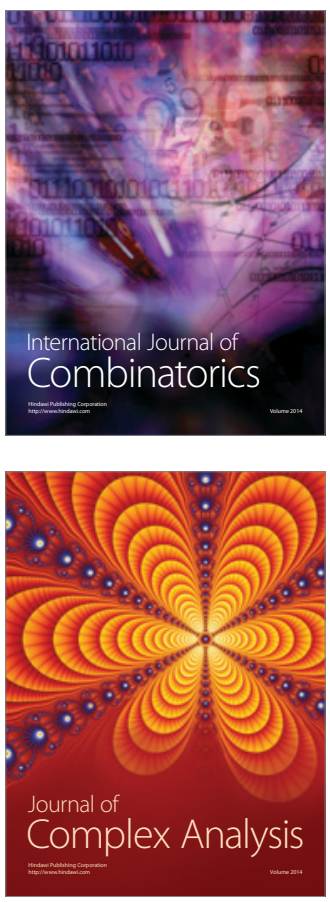

International Journal of

Mathematics and

Mathematical

Sciences
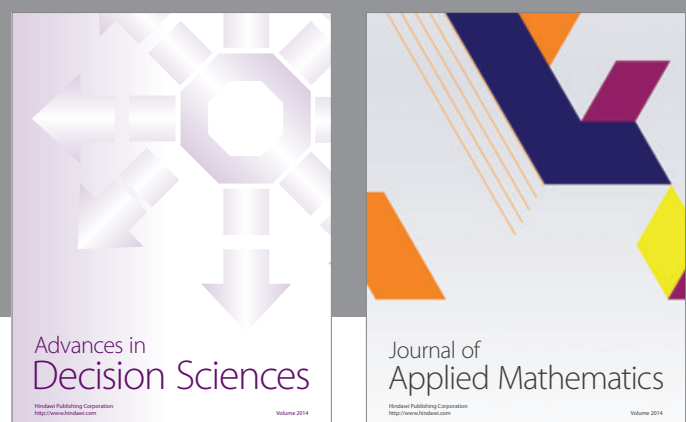

Journal of

Applied Mathematics
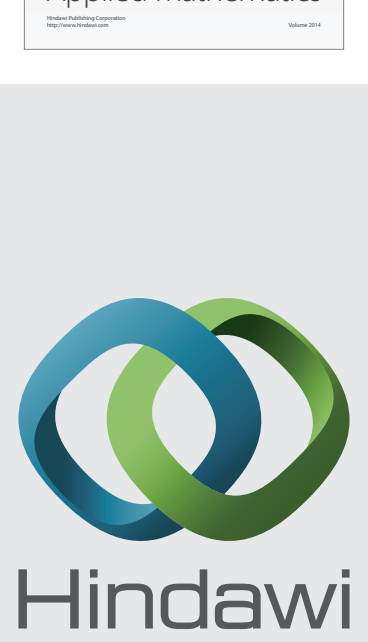

Submit your manuscripts at http://www.hindawi.com
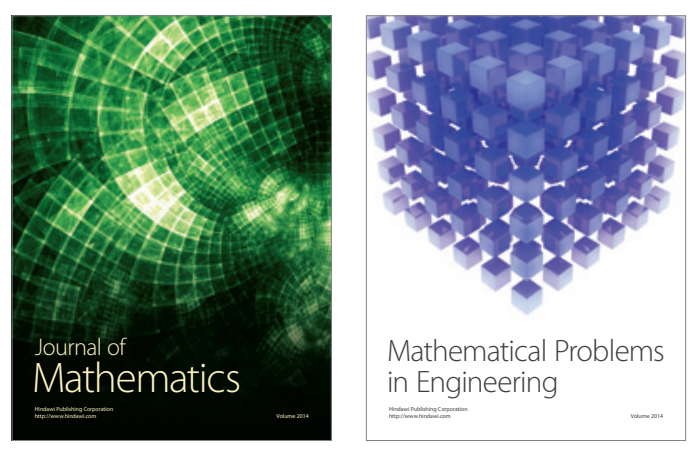

Mathematical Problems in Engineering
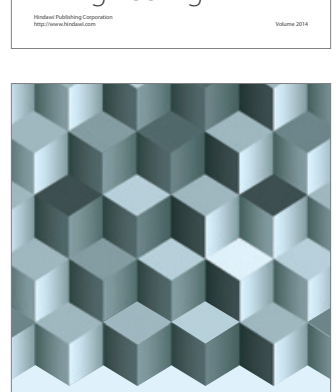

Journal of

Function Spaces
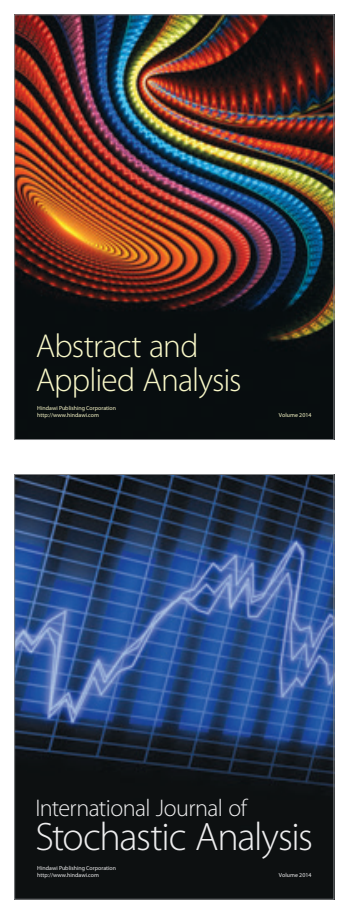

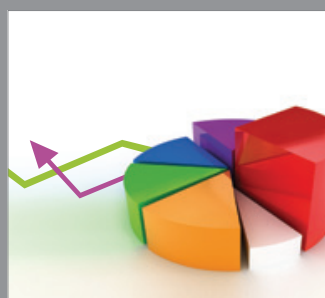

ournal of

Probability and Statistics

Promensencen
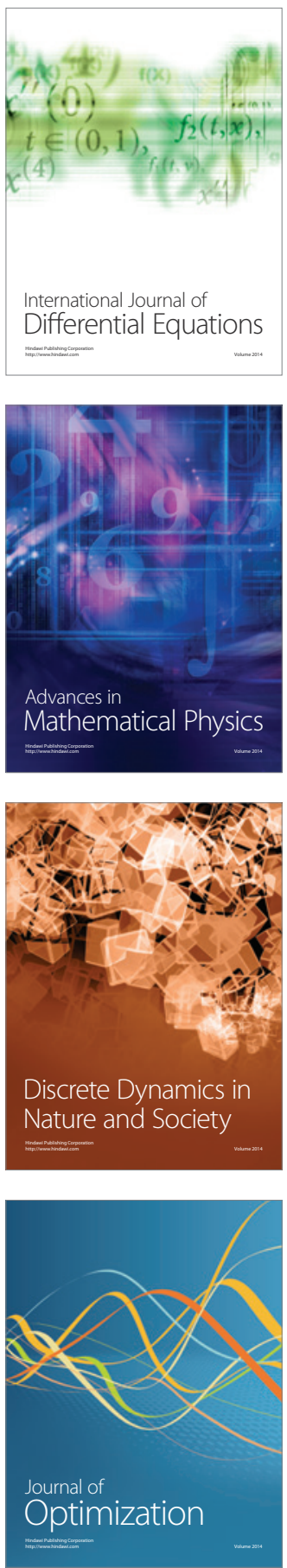